\title{
Binge Eating Disorder Mediates Links between Symptoms of Depression, Anxiety, and Caloric Intake in Overweight and Obese Women
}

\author{
Roseann E. Peterson, ${ }^{1,2}$ Shawn J. Latendresse, ${ }^{2,3}$ Lindsay T. Bartholome, ${ }^{4}$ \\ Cortney S. Warren, ${ }^{5}$ and Nancy C. Raymond ${ }^{4}$ \\ ${ }^{1}$ Department of Human and Molecular Genetics, Virginia Commonwealth University, Richmond, VA 23298, USA \\ ${ }^{2}$ Virginia Institute for Psychiatric and Behavioral Genetics, Virginia Commonwealth University, Biotechnology I, \\ Richmond, VA 23298, USA \\ ${ }^{3}$ Department of Psychiatry, Virginia Commonwealth University, Richmond, VA 23298, USA \\ ${ }^{4}$ Department of Psychiatry, University of Minnesota, Minneapolis, MN 55455, USA \\ ${ }^{5}$ Department of Psychology, University of Nevada, Las Vegas, NV 89154, USA
}

Correspondence should be addressed to Roseann E. Peterson, repeterson@vcu.edu

Received 31 December 2011; Revised 19 February 2012; Accepted 14 April 2012

Academic Editor: Kristin Schneider

Copyright (C) 2012 Roseann E. Peterson et al. This is an open access article distributed under the Creative Commons Attribution License, which permits unrestricted use, distribution, and reproduction in any medium, provided the original work is properly cited.

\begin{abstract}
Despite considerable comorbidity between mood disorders, binge eating disorder (BED), and obesity, the underlying mechanisms remain unresolved. Therefore, the purpose of this study was to examine models by which internalizing behaviors of depression and anxiety influence food intake in overweight/obese women. Thirty-two women (15 BED, 17 controls) participated in a laboratory eating-episode and completed questionnaires assessing symptoms of anxiety and depression. Path analysis was used to test mediation and moderation models to determine the mechanisms by which internalizing symptoms influenced kilocalorie (kcal) intake. The BED group endorsed significantly more symptoms of depression ( 10.1 versus $4.8, P=0.005)$ and anxiety ( 8.5 versus $2.7, P=0.003)$. Linear regression indicated that BED diagnosis and internalizing symptoms accounted for $30 \%$ of the variance in $\mathrm{kcal}$ intake. Results from path analysis suggested that BED mediates the influence of internalizing symptoms on total kcal intake (empirical $P<0.001$ ). The associations between internalizing symptoms and food intake are best described as operating indirectly through a BED diagnosis. This suggests that symptoms of depression and anxiety influence whether one engages in binge eating, which influences kcal intake. Greater understanding of the mechanisms underlying the associations between mood, binge eating, and food intake will facilitate the development of more effective prevention and treatment strategies for both BED and obesity.
\end{abstract}

\section{Introduction}

Although there is considerable comorbidity between obesity, eating disorders, and other major psychiatric disorders, the mechanisms underlying these associations have yet to be resolved. Binge eating disorder (BED), often associated with elevated body weight and mood disorders, is under consideration for inclusion in the Diagnostic and Statistical Manual of Mental Disorders, fifth edition (DSM-V). BED is defined by the DSM-IV as a provisional eating disorder diagnosis characterized by recurrent episodes of binge eating without weight control compensatory behavior and includes (1) "eating, in a discrete period of time (e.g., within any 2-hour period), an amount of food that is definitely larger than what most people would eat during a similar period of time and under similar circumstances" and (2) "a sense of lack of control over eating during the episode." In addition, individuals with BED must experience distress about their binge eating and endorse three of the following symptoms: eating more rapidly than normal, eating until uncomfortably full, eating large amounts when not hungry, eating alone because of embarrassment, and feeling disgusted, depressed, or guilty about overeating [1].

Although obesity is not a requirement for a BED diagnosis, research indicates that approximately $70 \%$ of those 
meeting criteria for BED are obese [2]. While the prevalence of BED in community samples ranges from $2-5 \%$, approximately $30 \%$ of obese individuals seeking weight control treatment meet criteria for BED $[3,4]$. The recurrent overeating that characterizes $\mathrm{BED}$, along with the absence of compensatory behaviors exhibited by those with bulimia nervosa (BN), is most likely responsible for the high frequency of obesity in this group. Laboratory studies have demonstrated that obese BED individuals consume significantly more kilocalories (kcal) during an overeating episode than obese individuals without a BED diagnosis [513].

Psychiatric disorders, including depression and anxiety, have been associated with obesity and BED. The lifetime prevalence of major depressive disorder (MDD) and anxiety disorders in the United States is estimated at $17 \%$ and $29 \%$, respectively [14]. However, within obese populations, reported lifetime prevalence rates are increased to $32.8 \%$ for depression and $30.5 \%$ for anxiety [15]. Additionally, Strine et al. found adults with a current or lifetime diagnosis of depression or anxiety were significantly more likely to engage in unhealthy behaviors such as physical inactivity and to be obese [15]. Furthermore, research shows that obese individuals with comorbid BED have even greater rates of depression and anxiety than obese individuals without BED [2, 16-19]. For example, Grilo et al. report, in a study of 404 BED patients, that lifetime history estimates were elevated to $52 \%$ for mood and $37.1 \%$ for anxiety disorders [17].

Despite general acknowledgment of the associations between bodyweight, BED, and comorbid psychiatric disorders, the mechanisms underlying these relationships remain largely unknown. Previously, we have reported that overweight/obese women with BED consume significantly greater kcal intake during a laboratory eating episode than weight-matched women without BED (2305 versus 1462 kcal) [13]. To extend this work, we assessed symptoms of depression and anxiety in this sample and sought to examine how internalizing behaviors and BED may be associated with kcal intake during the laboratory eating episode. Based on the literature, we hypothesized that participants meeting BED criteria would endorse significantly more symptoms of depression and anxiety than weight-matched non-BED controls. However, the impact of a BED diagnosis and symptoms of depression and anxiety on kcal intake was less clear as there are several potential mechanisms responsible for the association. It is possible that increased kcal intake is the result of BED symptomatology. For instance, those with BED may use binge eating to alleviate or escape symptoms of depression and anxiety. Additionally, in converse, it is possible that BED symptomatology such as distress regarding lack of control overeating specifically elevates internalizing symptoms. For example, depression may increase food intake through increased appetite, a clinical feature of atypical depression subtype. Furthermore, it is possible that having both a BED diagnosis and elevated symptoms of depression and anxiety synergistically influence food intake in a nonadditive manner.

A common statistical approach to examining relationships between variables is path analysis, in which alternative

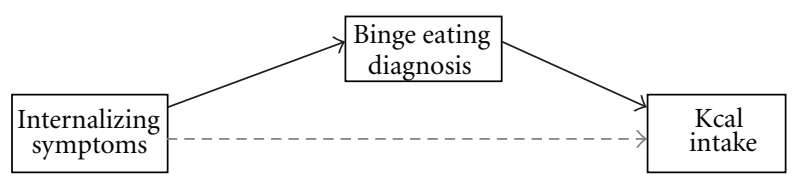

(a)

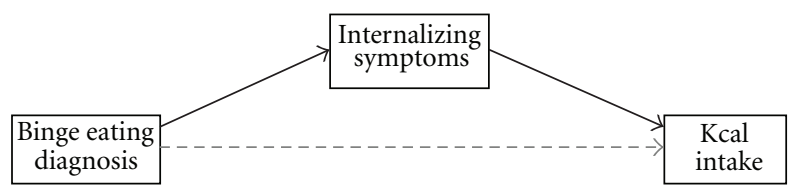

(b)

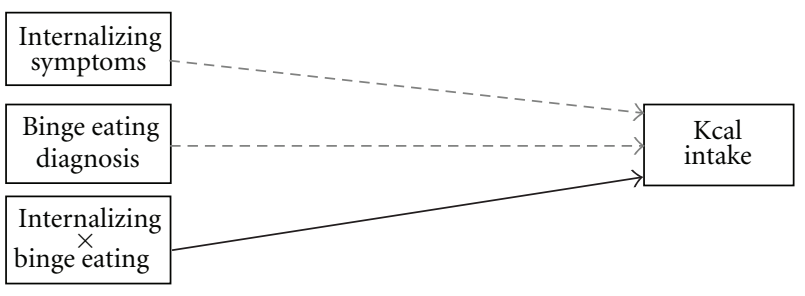

(c)

Figure 1: Theoretical models examined: (a) binge eating disorder mediates the associations between internalizing symptoms and kilocalorie intake, (b) internalizing symptoms mediate the association between binge eating disorder and kilocalorie intake, and (c) binge eating disorder interacts with internalizing symptoms in the prediction of kilocalorie intake. Note: internalizing: symptoms of depression and anxiety.

models can be applied to evaluate theoretical relationships and determine directionality of effects. We assessed three alternative models, depicted in Figure 1, to determine the mechanism of association that best fit our data. Path analysis was employed to evaluate three potential models: (1) the symptoms of depression and anxiety increase susceptibility to BED, which in turn influences caloric intake (Figure 1(a)), (2) a BED diagnosis influences symptoms of depression and anxiety, which subsequently influences caloric intake (Figure 1(b)) and (3) a BED diagnosis and symptoms of depression and anxiety function interdependently in relation to energy intake (Figure 1(c)).

\section{Methods}

2.1. Participants. Participants were recruited by newspaper and online advertisements inviting women at least 50 pounds overweight and between the ages of 18 and 45 to participate in a paid research study. Thirty-two women, including 15 meeting DSM-IV criteria for BED and 17 overweight/obese controls with no history of any binge eating or eating disorder behaviors, participated in the study. These women were recruited as part of a larger study examining food intake and energy expenditure measured via the doubly labeled water method $[13,20]$.

2.2. Group Assignment. Potential participants were interviewed with the Structured Clinical Interview for DSMIV Axis I Disorders, Patient Edition (SCID-I/P) [21], and 
the Eating Disorder Examination (EDE), Version 12.0D [22] to determine study eligibility and group assignment. Additionally, a medical history, physical exam, and battery of laboratory tests were completed to detect unstable medical conditions, such as diabetes and impaired thyroid function, which would influence eligibility. Participants were excluded from the study if they had any unstable medical or psychiatric conditions, met DSM-IV criteria for substance abuse or dependency within 6 months of participation, or were currently dieting or participating in a weight-loss program. Those with any history of $\mathrm{BN}$ or compensatory behaviors were also excluded. Non-BED controls were free of any current or past eating disorder symptoms. The protocol was reviewed and approved by the Institutional Review Board at the University of Minnesota, and all participants took part in the informed consent process and signed a consent form. Participants were paid $\$ 300$ upon completion of the entire study protocol.

2.3. Laboratory Binge Eating Episode. This study utilized a protocol our group has previously reported [12, 13, 23]. In brief, participants were interviewed by a research dietician regarding their general eating patterns and foods on which they typically snacked or overate. They indicated which items from a standardized list of snack foods appealed to them and could suggest extra foods or recipes. Based on the information gathered during the interview, a tray of binge foods was created for each participant incorporating their personalized snacking preferences. Each participant received 6 to 10 different kinds of food on their snack tray. Food items were presented in excessive quantities (two to three times what they endorsed eating during a binge) to ensure that binge size was not limited by quantity of food.

Participants were admitted to the General Clinical Research Center (GCRC) for an overnight stay to participate in several study activities. They were instructed not to consume any food or caloric beverages between 12 and 5 PM. At approximately 5:30 PM, they were presented with a multiple-item array of foods, including their personalized binge tray and a standard hospital dinner and were instructed to "Let yourself go and eat as much as you like." They were left alone in a private room to eat for as long as they liked and signaled the nursing staff when they were finished. The GCRC metabolic kitchen staff measured pre- and postprandial quantities of food. Caloric and macronutrient intake for the laboratory eating episodes were calculated using Nutritionist IV [24].

2.4. Self-Report Measures of Depression and Anxiety. During the initial evaluation, participants completed the Beck Depression Inventory (BDI) and the Beck Anxiety Inventory (BAI) which are widely used self-report questionnaires consisting of items addressing how one has been feeling in the last week and measure the severity of depression and anxiety symptoms $[25,26]$. The scales have high internal consistency coefficients (i.e., BDI upwards of 0.80), and validity with other clinical assessments $[27,28]$. Scores on these indices range from 0 to 63 and correspond to normal
(0-9 BDI, 0-7 BAI), mild (10-18 BDI, 8-15 BAI), moderate (19-29 BDI, 16-25 BAI), and severe (30-63 BDI, 26-63 BAI) depression and anxiety.

2.5. Analytic Strategy and Model Validation. A set of three, theoretically driven path models (see Figure 1) were tested using Mplus version 5.0 [29]. As MacKinnon and colleagues [30] have suggested, the traditional causal steps approach [31, 32] may lack the statistical power to detect some meaningful indirect effects. The mediation analyses presented here utilized the product of coefficients strategy $[30,33]$ to evaluate the extent to which a predictor influences an outcome through some intermediary variable (Figures $1(\mathrm{a})$ and $1(\mathrm{~b})$ ). In doing so, the indirect effect is derived by taking a ratio of the product of the path coefficients from (1) the independent variable to the mediator and (2) the mediator to the dependent variable over the normal-theory standard error for that product (i.e., $\left.(\beta 1 * \beta 2) / \mathrm{SE}_{(\beta 1 * \beta 2)}\right)$, the results of which are evaluated with respect to the $Z$-distribution. Moderation (Figure 1(c)) was assessed via the partial path coefficient for a product term (i.e., $\beta / \mathrm{SE}_{\beta}$ ) in the presence of its individual components and evaluated with respect to a $t$-distribution.

To protect against potential bias introduced by the small size of our sample, evidence of significance was assessed via permutation testing [34]. From the originally observed data, ten thousand novel datasets were generated via the random reordering of individuals' values on BED and kcal intake. This procedure was performed in R version 2.9.1 [35]. Each of the permuted datasets can thus be reanalyzed within Mplus version 5.0 [29], with respect to the three alternative models depicted in Figure 1, and the test statistics from each iteration can be used to generate null distributions for each of the effects being scrutinized. Criteria for significance (i.e., empiric $P$ values) can be calculated using the formula $(p+1) /(n+1)$, where $p$ is the number of null tests that are more significant than the test conducted with the original data and $\mathrm{n}$ is the total number of permutations (i.e., 10,000) on which the analyses are rerun. As a result, we are able to assess whether each of the hypothesized models would achieve significance in a much larger sample (i.e., 320,000), given the characteristics of our observed sample.

\section{Results}

3.1. Descriptive Statistics. Of the thirty-two women participants, 27 were European-American, 3 were AfricanAmerican (9.4\%), and 2 were Asian-American (6.3\%). Means and standard deviations for total energy intake, depression and anxiety scores, and potential covariates (i.e., age and $\mathrm{BMI}$ ) are presented by BED diagnosis on the diagonal in Table 1. ANOVA indicated that there were significant group differences in depression scores (10.1 versus 4.8, $F(1,30)=9.308, P=0.005)$ and anxiety scores (8.5 versus $2.7, F(1,30)=10.830, P=0.003)$ with $\mathrm{BED}$ participants having significantly higher mean scores than controls across these indices. No intergroup differences were found regarding $\operatorname{BMI}(F(1,30)=3.203, P=0.784)$, or age $(F(1,30)=10.737, P=0.674)$. Table 2 reports 
the prevalence of lifetime clinical depression and anxiety diagnoses by group. The BED group had significantly greater prevalence of mild depression (60 versus $17.6 \%, \chi^{2}=6.10$, $P=0.014)$, mild $/$ moderate anxiety (33.3 versus $5.9 \%, \chi^{2}=$ $3.94, P=0.047$ ), and anxiety disorders (46.7 versus $11.8 \%$, $\chi^{2}=4.80, P=0.028$ ). A detailed examination of food intake and energy expenditure in these participants is reported in additional manuscripts from our group $[13,20]$.

Pearson's correlation coefficients for bivariate associations between study variables are presented in the offdiagonal cells in Table 1 . Within each cell, associations are presented separately for participants diagnosed with BED (top), weight-matched controls (middle), and across the entire sample (bottom). Significant positive correlations were found between kcal intake and depression, kcal intake and anxiety, and depression and anxiety within the full sample. When assessed within groups, no significant correlations were found except between depression and anxiety scales in the control group. Since neither BED nor internalizing symptoms were associated with age or BMI, these latter variables were not included in the path models described below.

3.2. Model Fitting. Separate path models were run to test (a) the intermediary role of BED in associations between depression and anxiety symptoms and caloric intake, (b) the intermediary role of depression and anxiety symptoms in associations between BED and caloric intake, and (c) the interactive influences of BED and symptoms of depression and anxiety on caloric intake. In each case, the theoretical model accounted for a significant amount ( $30 \%)$ of the variance in energy intake. However, examination of the three alternative theoretical models revealed important mechanistic differences in the relationship between BED and symptoms of depression and anxiety as they serve to jointly influence energy intake. Results of the models (Table 3) depicted in Figures 1(a) and 1(b) suggest that, while kcal intake is significantly influenced by both depression and anxiety symptoms $\left(\beta_{\text {total, depression }}=0.409\right.$, $\left.P=0.006 ; \beta_{\text {total, anxiety }}=0.399, P=0.003\right)$ and binge eating status $\left(\beta_{\text {total, } \mathrm{BED}}=-0.508, P \leq 0.001\right)$, the effects attributed to symptoms of depression and anxiety operate, in large part, through the influences they have on BED ( $\beta_{\text {indirect, depression via BED }}=0.197, P=0.052$; $\left.\beta_{\text {indirect, anxiety via } \mathrm{BED}}=0.212, P=0.046\right)$. Note that the sign of the effects reflect coding of 1 for BED and 2 for controls in all analyses. That is, roughly half of the influence of depression $(\sim 48 \%)$ and anxiety $(\sim 53 \%)$ on caloric intake is mediated through BED. In contrast, the influence of BED status on caloric intake appears not to be mediated by symptoms of depression or anxiety $\left(\beta_{\text {indirect, } B E D \text { via depression }}=-0.103, P=\right.$ $\left.0.282 ; \beta_{\text {indirect, } \mathrm{BED} \text { via anxiety }}=-0.096, P=0.329\right)$; rather, those direct effects remained strong $\left(\beta_{\text {direct, } B E D \text { with depression }}=\right.$ $-0.404, P=0.027 ; \beta_{\text {direct, }}$ BED with anxiety $=-0.411, P=$ 0.014). Results of the model depicted in Figure 1(c) indicate that BED and symptoms of depression and anxiety do not interdependently influence caloric intake, that is, neither the model including depression nor the model including anxiety yielded significant partial path coefficients for an interaction between BED and the corresponding depression or anxiety symptoms $\left(\beta_{\mathrm{BED} x \text { depression }}=-0.279, P=0.598 ; \beta_{\mathrm{BED} x \text { anxiety }}=\right.$ $-0.268, P=0.609)$ after taking into account their combined main effects, in each case accounting for less than $1 \%$ of the total variance.

As described above, post hoc analyses were conducted with 10,000 permuted datasets to determine whether the results observed with respect to the first theoretical model (i.e., BED mediating the association between symptoms of depression and anxiety and caloric intake) were simply due to chance and/or an artifact of the modest size of the present sample. The null distributions generated from these analyses suggested that the indirect effects of both depression and anxiety through BED were highly significant; as far fewer than $5 \%$ of the tests exceeded the $P$ values observed in the original data. In fact, of the 10,000 randomly generated datasets, only seven yielded indirect effects of depression through $\mathrm{BED}$ that were more significant than the effect observed in the original data $(P=0.0008)$, with only thirty-eight indirect effects of anxiety on BED exceeding the observed level of significance $(P=0.0009)$.

\section{Discussion}

The purpose of this study was to examine models by which internalizing symptoms of depression and anxiety influence food intake in overweight/obese women. Our results indicate that BED women endorse significantly more symptoms of depression and anxiety. Additionally, linear regression indicated that $\mathrm{BED}$ diagnosis and internalizing symptoms accounted for $30 \%$ of the variance in kcal intake. Furthermore, results from path analysis imply that BED mediates the influence of internalizing symptoms on total kcal-intake, which suggests the associations between internalizing symptoms and food intake are best described as operating indirectly through a BED diagnosis.

The present study found that overweight/obese women with BED endorsed more symptoms of depression and anxiety than non-BED weight-matched controls. Mean scores for the BDI and the BAI indicated mild depression and anxiety in the BED group but normal levels in the control group. Other studies have found elevated depression and anxiety scores in BED individuals $[5,9,18,19,36,37]$. For example, in a study by Fandiño et al., depression and anxiety scores were significantly greater in the BED group than those in the obese control group as assessed by the Symptom Checklist 90 and the BDI [37]. The lifetime prevalence of MDD in the BED and control groups was $46.7 \%$ and $29.4 \%$, respectively. These rates are similar to previous reports in $\operatorname{BED}[2,16-$ 19] and non-BED obese groups [15]. Lifetime prevalence of anxiety disorders was similar to rates of depression in the BED group (46.7\%) but was much lower in the control group $(11.8 \%)$. It is possible that the lower rates of anxiety disorders in the control group were due to the inclusion of overweight women or was an artifact of the limited sample size.

Laboratory studies have demonstrated that those with BED have greater total food intake than obese controls 
TABLE 1: Group means and intercorrelations for study variables.

\begin{tabular}{|c|c|c|c|c|c|}
\hline & 1 & 2 & 3 & 4 & 5 \\
\hline \multirow{2}{*}{ (1) Age } & $30.1(6.7)$ & & & & \\
\hline & $31.3(8.5)$ & & & & \\
\hline \multirow{3}{*}{ (2) Body mass index $\left(\mathrm{kg} / \mathrm{m}^{2}\right)$} & -0.17 & $34.3(5.5)$ & & & \\
\hline & -0.14 & $34.9(7.2)$ & & & \\
\hline & -0.15 & & & & \\
\hline \multirow{3}{*}{ (3) Depression symptoms } & -0.09 & -0.33 & $10.1(4.8)$ & & \\
\hline & 0.06 & 0.34 & $4.8(5.0)$ & & \\
\hline & -0.04 & 0.04 & & & \\
\hline \multirow{3}{*}{ (4) Anxiety symptoms } & -0.05 & -0.29 & 0.34 & $8.5(6.5)$ & \\
\hline & 0.35 & 0.24 & $0.66^{* *}$ & $2.7(3.1)$ & \\
\hline & 0.04 & -0.08 & $0.57 * * *$ & & \\
\hline \multirow{3}{*}{ (5) Kilocalorie intake } & -0.10 & -0.06 & 0.28 & 0.27 & $2305.1(834.0)$ \\
\hline & -0.26 & $0.54^{*}$ & 0.15 & 0.00 & $1461.8(641.9)$ \\
\hline & -0.19 & 0.02 & $0.41^{*}$ & $0.40^{*}$ & \\
\hline
\end{tabular}

${ }^{*} P<0.05 ;{ }^{* *} P<0.01 ; * * * P<0.001$.

Notes: off-diagonal cells depict Pearson's correlation coefficients for participants diagnosed with binge eating disorder (top; $n=15)$, controls (middle; $n=$ 17), and the overall sample (bottom; $n=32$ ); values on the diagonal reflect means and standard deviations for cases (top) and controls (bottom), with boldface type indicating group differences $(P<0.01)$ as assessed via $F$-statistic with 1,30 degrees of freedom.

TABLE 2: Lifetime clinical depression and anxiety diagnoses by group.

\begin{tabular}{|c|c|c|c|c|}
\hline Diagnosis & BED $n(\%)$ & Control $n(\%)$ & Chi-square & $P$ value \\
\hline BDI-mild & $9(60 \%)$ & $3(17.6 \%)$ & 6.10 & 0.014 \\
\hline BAI-mild/moderate & $5(33.3 \%)$ & $1(5.9 \%)$ & 3.94 & 0.047 \\
\hline $\mathrm{MDD}$ & $7(46.7 \%)$ & $5(29.4 \%)$ & 1.01 & 0.314 \\
\hline Dep NOS & $1(6.7 \%)$ & $0(0 \%)$ & 1.17 & 0.279 \\
\hline GAD & $1(6.7 \%)$ & $0(0 \%)$ & 1.17 & 0.279 \\
\hline Social phobia & $4(26.7 \%)$ & $1(5.9 \%)$ & 2.61 & 0.106 \\
\hline Specific phobia & $2(13.3 \%)$ & $0(0 \%)$ & 2.42 & 0.120 \\
\hline Panic disorder & $1(6.7 \%)$ & $0(0 \%)$ & 1.17 & 0.279 \\
\hline PTSD & $1(6.7 \%)$ & $0(0 \%)$ & 1.17 & 0.279 \\
\hline Anx NOS & $0(0 \%)$ & $2(11.8 \%)$ & 1.88 & 0.170 \\
\hline Any Dep Dx & $8(53.3 \%)$ & $5(29.4 \%)$ & 1.89 & 0.169 \\
\hline Any Anx Dx & $7(46.7 \%)$ & $2(11.8 \%)$ & 4.80 & 0.028 \\
\hline Any Dep/Anx Dx & $10(66.7 \%)$ & $6(35.3 \%)$ & 3.14 & 0.077 \\
\hline
\end{tabular}

Note: BED: binge eating disorder, Chi-square: Pearson's Chi-square 1 degree of freedom test, BDI-mild: mild depression as assessed by the Beck Depression Inventory which corresponds to scores 10-18, BAI-mild/moderate: mild to moderate anxiety as assessed by the Beck Anxiety Inventory which corresponds to scores 8-25, MDD: major depressive disorder, Dep NOS: depressive disorder not otherwise specified, PTSD: posttraumatic stress disorder, Anx NOS: anxiety disorder not otherwise specified, any Dep Dx: any DSM-IV depressive disorder diagnosis, any Anx Dx: any DSM-IV anxiety disorder diagnosis, any Dep/Anx Dx: any DSM-IV depressive or anxiety disorder diagnosis, dysthymic disorder, and obsessive compulsive disorder were omitted from table because no participants met criteria for these disorders.

TABle 3: Standardized effects coefficients, standard errors, and corresponding $P$ values for mediation models.

\begin{tabular}{|c|c|c|c|c|c|c|c|c|c|c|c|}
\hline \multirow{2}{*}{ Predictor } & \multirow{2}{*}{ Mediator } & \multicolumn{3}{|c|}{ Total effect } & \multicolumn{3}{|c|}{ Direct effect } & \multicolumn{4}{|c|}{ Indirect (mediated) effect } \\
\hline & & $\beta$ & SE & $P$ value ${ }^{\mathrm{a}}$ & $\beta$ & SE & $P$ value ${ }^{\mathrm{a}}$ & $\beta$ & SE & $P$ value ${ }^{\mathrm{a}}$ & Empirical $P$ value ${ }^{\mathrm{b}}$ \\
\hline \multicolumn{12}{|l|}{ Model 1} \\
\hline Depression & BED & 0.409 & 0.150 & 0.006 & 0.212 & 0.183 & 0.247 & 0.197 & 0.101 & 0.052 & 0.0008 \\
\hline Anxiety & $\mathrm{BED}$ & 0.399 & 0.136 & 0.003 & 0.187 & 0.181 & 0.301 & 0.212 & 0.106 & 0.046 & 0.0009 \\
\hline \multicolumn{12}{|l|}{ Model 2} \\
\hline BED & Depression & -0.508 & 0.136 & $<0.001$ & -0.404 & 0.182 & 0.027 & -0.103 & 0.096 & 0.282 & - \\
\hline BED & Anxiety & -0.508 & 0.136 & $<0.001$ & -0.411 & 0.168 & 0.014 & -0.096 & 0.099 & 0.329 & - \\
\hline
\end{tabular}

${ }^{a}$ Corresponding to the two-tailed test statistics for models run with sample data.

${ }^{b}$ Corresponding to the two-tailed test statistics for a series of analyses with 10,000 permuted datasets.

Note: BED: binge eating disorder, signs of effects reflect coding of BED status as 1 and control as 2 in all analyses. 
when instructed to overeat [5-13]. Two such studies have reported on both food intake and depression symptoms $[5,9]$. In a sample of 10 obese BED women and 9 obese controls, Yanovski et al. found that the BED group consumed significantly more kcals (2962 versus 2017) and had significantly greater depression scores as measured by the BDI (18.9 versus 5.4) than controls. Additionally, they observed significant positive correlations between kcal intake and BDI score $\left(r^{2}=0.41\right)$ and between binge meal energy intake and BDI $\left(r^{2}=0.28\right)$. Geliebter and colleagues compared consumption of a liquid test-meal for 30 obese BED individuals ( 18 women) and 55 obese controls (43 women). The BED group consumed significantly more grams $(1,032$ versus 737) of the liquid test meal and endorsed significantly higher depression scores assessed by the Zung Depression Scale. However, a significant correlation between test meal intake and depression score was not found. The discrepancy could be due to several study design differences, including proportion of BED and control participants, inclusion of men, and type of food intake (solid versus liquid meal).

Furthermore, results from linear regression indicated that BED diagnosis and symptoms of depression and anxiety accounted for a significant amount $(\sim 30 \%)$ of the variance in caloric intake. However, examination of the three alternative models revealed important mechanistic differences in the relationship between $\mathrm{BED}$, symptoms of depression, and anxiety and subsequent energy intake. The model that best fit our data indicated that BED mediated the influence of depression and anxiety symptoms on total kcal intake (Figure 1(a)). Specifically, our results suggest that the associations found between symptoms of depression and anxiety and food intake are best described as operating indirectly through a BED diagnosis, that is, symptoms of depression and anxiety influence whether one engages in pathological binge eating, which, in turn, influences caloric intake. Our findings did not support model b (BED predicted symptoms of depression and anxiety which, in turn, influence kcal intake), or model c (a significant interaction between symptoms of depression and anxiety and BED as being predictive of kcal intake).

These results highlight the importance of mood in relation to a BED diagnosis and subsequent caloric intake. Other research has also implicated mood in BED. Telch et al. interviewed 60 obese women with BED regarding their definition of binge eating, and 33\% reported it as eating to regulate negative affect [38]. With the advent of the ecological momentary assessment procedures (EMA), prospective data on precursors to binge eating in the natural environment have been collected [39-43]. A study by Stein et al. found in 33 obese women with BED that negative mood was significantly greater at prebinge times than at nonbinge times and that participants attributed binge eating to mood more frequently than hunger or violation of extreme dietary restraint (abstinence violation) [41]. Additionally, a study by Hilbert and Tuschen-Caffier found that mood preceding a binge eating episode was more negative than mood prior to regular eating or at random assessments in a sample of 20 obese women with BED [42]. Furthermore, in a meta-analysis of 36 EMA studies of $\mathrm{BED}$ and $\mathrm{BN}$, negative affect was significantly greater preceding binge eating relative to average affect and affect before regular eating [43]. A growing body of literature implicates negative affect as a precursor to binge eating in BED.

The implications of the present study are potentially relevant to the clinical treatment of BED and obesity. Research has indicated that mood and eating disorder diagnoses affect weight loss, and other treatment efforts. For example, Pagoto et al. reported that both BED and depression were associated with less weight loss and depression was associated with study attrition [44]. Furthermore, in BED treatment, depression symptoms have been associated with both attrition from cognitive-behavioral therapy and severity of eating disorder psychopathology [45]. The current results suggest that targeting mood may be useful in the treatment of BED and accentuate the importance of considering mood and BED status in weight management.

Among the major strengths of this study were utilizing path analysis to test relationships between BED, symptoms of depression and anxiety, and kcal intake as well as using permutation procedures for model validation and statistical support. EMA studies have consistently demonstrated negative affect as a precursor to binge eating [43] in BED. However, these studies have relied on self-report of food intake. Research indicates that obese and BED populations tend to underreport their food intake $[13,20$, 46-51]. Therefore, a further strength of this work was the inclusion of laboratory-measured food intake to avoid inaccuracies often associated with self-report of dietary intake. Potential limitations include limited sample size and age range, exclusion of male participants, and use of selfreport questionnaires to measure symptoms of depression and anxiety. Future research is warranted to confirm our findings and should seek to compare energy intake and depression and anxiety in both women and men. Greater understanding of the mechanisms underlying the associations of depression and anxiety symptoms, binge eating, and caloric intake will facilitate the development of more effective prevention and treatment strategies for both BED and obesity.

\section{Conflict of Interests}

The authors declare no conflict of interest.

\section{Acknowledgments}

This study was funded by the National Institutes of Health Grants (R01 MH 060199, MO1-RR00400), supported in part by the Minnesota Obesity Center Grant (P30 DK 60456) and the National Institute on Drug Abuse (DA-26119). Special thanks to Brion S. Maher, Ph.D., for statistical consultation on permutation procedures and Jennifer Hommerding, Psy.D., and Andrea Loveless, Psy.D., for assistance with data collection. 


\section{References}

[1] American Psychiatric Association, Ed., Diagnostic and Statistical Manual of Mental Disorders, Washington, DC, USA, 4th edition, 1994.

[2] R. A. Grucza, T. R. Przybeck, and C. R. Cloninger, "Prevalence and correlates of binge eating disorder in a community sample," Comprehensive Psychiatry, vol. 48, no. 2, pp. 124-131, 2007.

[3] R. L. Spitzer, S. Yanovski, T. Wadden et al., "Binge eating disorder: its further validation in a multisite study," International Journal of Eating Disorders, vol. 13, no. 2, pp. 137-153, 1993.

[4] M. de Zwaan, "Binge eating disorder and obesity," International Journal of Obesity, vol. 25, supplement 1, pp. S51-S55, 2001.

[5] S. Z. Yanovski, M. Leet, J. A. Yanovski et al., "Food selection and intake of obese women with binge-eating disorder," American Journal of Clinical Nutrition, vol. 56, no. 6, pp. 975980, 1992.

[6] J. A. Goldfein, B. T. Walsh, J. L. LaChaussee, H. R. Kissileff, and M. J. Devlin, "Eating behavior in binge eating disorder," International Journal of Eating Disorders, vol. 14, no. 4, pp. 427-431, 1993.

[7] E. A. Cooke, J. L. Guss, H. R. Kissileff, M. J. Devlin, and B.T. Walsh, "Patterns of food selection during binges in women with binge eating disorder," International Journal of Eating Disorders, vol. 22, no. 2, pp. 187-193, 1997.

[8] B. A. Gosnell, J. E. Mitchell, K. L. Lancaster, M. A. Burgard, S. A. Wonderlich, and R. D. Crosby, "Food presentation and energy intake in a feeding laboratory study of subjects with binge eating disorder," International Journal of Eating Disorders, vol. 30, no. 4, pp. 441-446, 2001.

[9] A. Geliebter, G. Hassid, and S. A. Hashim, "Test meal intake in obese binge eaters in relation to mood and gender," International Journal of Eating Disorders, vol. 29, no. 4, pp. 488-494, 2001.

[10] J. L. Guss, H. R. Kissileff, M. J. Devlin, E. Zimmerli, and B. T. Walsh, "Binge size increases with body mass index in women with binge-eating disorder," Obesity Research, vol. 10, no. 10, pp. 1021-1029, 2002.

[11] N. C. Raymond, B. Neumeyer, C. S. Warren, S. S. Lee, and C. B. Peterson, "Energy intake patterns in obese women with binge eating disorder," Obesity Research, vol. 11, no. 7, pp. 869-879, 2003.

[12] L. T. Bartholome, N. C. Raymond, S. S. Lee, C. B. Peterson, and C. S. Warren, "Detailed analysis of binges in obese women with binge eating disorder: comparisons using multiple methods of data collection," International Journal of Eating Disorders, vol. 39, no. 8, pp. 685-693, 2006.

[13] L. T. Bartholome, R. E. Peterson, S. K. Raatz, and N. C. Raymond, "A comparison of the accuracy of self-reported intake with measured intake of a laboratory overeating episode in overweight and obese women with and without binge eating disorder," European Journal of Nutrition. In press.

[14] R. C. Kessler, W. T. Chui, O. Demler, and E. E. Walters, "Prevalence, severity, and comorbidity of 12-month DSM-IV disorders in the National Comorbidity Survey Replication," Archives of General Psychiatry, vol. 62, no. 6, pp. 617-627, 2005.

[15] T. W. Strine, A. H. Mokdad, S. R. Dube et al., "The association of depression and anxiety with obesity and unhealthy behaviors among community-dwelling US adults," General Hospital Psychiatry, vol. 30, no. 2, pp. 127-137, 2008.
[16] M. de Zwaan, J. E. Mitchell, H. C. Seim et al., "Eating related and general psychopathology in obese females with binge eating disorder," International Journal of Eating Disorders, vol. 15, no. 1, pp. 43-52, 1994.

[17] C. M. Grilo, M. A. White, and R. M. Masheb, "DSM-IV psychiatric disorder comorbidity and its correlates in binge eating disorder," International Journal of Eating Disorders, vol. 42, no. 3, pp. 228-234, 2009.

[18] L. Azarbad, J. Corsica, B. Hall, and M. Hood, "Psychosocial correlates of binge eating in Hispanic, African American, and Caucasian women presenting for bariatric surgery," Eating Behaviors, vol. 11, no. 2, pp. 79-84, 2010.

[19] L. Jones-Corneille, T. Wadden, D. Sarwer et al., "Axis I psychopathology in bariatric surgery candidates with and without binge eating disorder: results of structured clinical interviews," Obesity Surgery, vol. 22, no. 3, pp. 389-397, 2012.

[20] N. Raymond, R. Peterson, L. Bartholome, S. Raatz, M. Jensen, and J. Levine, "Comparisons of energy intake and energy expenditure in overweight and obese women with and without binge eating disorder," Obesity, vol. 20, no. 4, pp. 765-772, 2012.

[21] M. B. First, R. L. Spitzer, M. Gibbon, and J. B. W. Williams, Structured Clinical Interview for DSM-IV Axis I Disorders. Patient Edition (SCID-P, Version 2), New York State Psychiatric Institute, Biometrics Research, New York, NY, USA, 1995.

[22] C. G. Fairburn and Z. Cooper, The Eating Disorder Examination, Guilford, New York, NY, USA, 1993.

[23] N. C. Raymond, L. T. Bartholome, S. S. Lee, R. E. Peterson, and S. K. Raatz, "A comparison of energy intake and food selection during laboratory binge eating episodes in obese women with and without a binge eating disorder diagnosis," International Journal of Eating Disorders, vol. 40, no. 1, pp. 67-71, 2007.

[24] Nutritionist IV, Hearst Corporation, San Burno, Calif, USA, 1999.

[25] A. T. Bcek, C. H. Ward, M. Mendelson, J. Mock, and J. Erbaugh, "An inventory for measuring depression," Archives of General Psychiatry, vol. 4, pp. 561-571, 1961.

[26] A. T. Beck, N. Epstein, G. Brown, and R. A. Steer, "An inventory for measuring clinical anxiety: psychometric properties," Journal of Consulting and Clinical Psychology, vol. 56, no. 6, pp. 893-897, 1988.

[27] M. A. Stanley and J. G. Beck, "Anxiety disorders," Clinical Psychology Review, vol. 20, no. 6, pp. 731-754, 2000.

[28] A. T. Beck, R. A. Steer, and M. G. Garbin, "Psychometric properties of the Beck Depression Inventory: twenty-five years of evaluation," Clinical Psychology Review, vol. 8, no. 1, pp. 77100, 1988.

[29] L. K. Muthén and B. O. Muthén, Mplus User's Guide, version 5.0, 5th edition, 2007.

[30] D. MacKinnon, C. Lockwood, J. Hoffman, S. G. West, and V. Sheets, "A comparison of methods to test mediation and other intervening variable effects," Psychological Methods, vol. 7, no. 1, pp. 83-104, 2002.

[31] R. M. Baron and D. A. Kenny, "The moderator-mediator variable distinction in social psychological research. Conceptual, strategic, and statistical considerations," Journal of Personality and Social Psychology, vol. 51, no. 6, pp. 1173-1182, 1986.

[32] C. M. Judd and D. A. Kenny, "Process analysis," Evaluation Review, vol. 5, no. 5, pp. 602-619, 1981.

[33] K. Preacher and A. Hayes, "SPSS and SAS procedures for estimating indirect effects in simple mediation models," Behavior Research Methods, Instruments, and Computers, vol. 36, no. 4, pp. 717-731, 2004. 
[34] P. Armitage and T. Colton, Encyclopedia of Biostatistics, John Wiley \& Sons, Hoboken, NJ, USA, 2005.

[35] R Development Core Team, R: A Language and Environment for Statistical Computing, version 2.9.2., 2009.

[36] C. Peterson, P. Thuras, D. Ackard et al., "Personality dimensions in bulimia nervosa, binge eating disorder, and obesity," Comprehensive Psychiatry, vol. 51, no. 1, pp. 31-36, 2010.

[37] J. Fandiño, R. Moreira, C. Preissler et al., "Impact of binge eating disorder in the psychopathological profile of obese women," Comprehensive Psychiatry, vol. 51, no. 2, pp. 110-114, 2010.

[38] C. F. Telch, E. M. Pratt, and S. H. Niego, "Obese women with binge eating disorder define the term binge," International Journal of Eating Disorders, vol. 24, no. 3, pp. 313-317, 1998.

[39] J. Smyth, S. Wonderlich, M. Sliwinski et al., "Ecological momentary assessment of affect, stress, and binge-purge behaviors: day of week and time of day effects in the natural environment," International Journal of Eating Disorders, vol. 42, no. 5, pp. 429-436, 2009.

[40] J. Smyth, S. Wonderlich, R. Crosby, R. Miltenberger, J. Mitchell, and M. Rorty, "The use of ecological momentary assessment approaches in eating disorder research," International Journal of Eating Disorders, vol. 30, no. 1, pp. 83-95, 2001.

[41] R. Stein, J. Kenardy, C. Wiseman, J. Dounchis, B. Arnow, and D. Wilfley, "What's driving the binge in binge eating disorder? A prospective examination of precursors and consequences," International Journal of Eating Disorders, vol. 40, no. 3, pp. 195-203, 2007.

[42] A. Hilbert and B. Tuschen-Caffier, "Maintenance of binge eating through negative mood: a naturalistic comparison of binge eating disorder and bulimia nervosa," International Journal of Eating Disorders, vol. 40, no. 6, pp. 521-530, 2007.

[43] A. Haedt-Matt and P. Keel, "Revisiting the affect regulation model of binge eating: a meta-analysis of studies using ecological momentary assessment," Psychological Bulletin, vol. 137, no. 4, pp. 660-681, 2011.

[44] S. Pagoto, J. Bodenlos, L. Kantor, M. Gitkind, C. Curtin, and Y. Ma, "Association of major depression and binge eating disorder with weight loss in a clinical setting," Obesity, vol. 15, no. 11, pp. 2557-2559, 2007.

[45] R. M. Masheb and C. M. Grilo, "Examination of predictors and moderators for self-help treatments of binge-eating disorder," Journal of Consulting and Clinical Psychology, vol. 76, no. 5, pp. 900-904, 2008.

[46] A. M. Prentice, A. E. Black, W. A. Coward et al., "High levels of energy expenditure in obese women," British Medical Journal, vol. 292, no. 6526, pp. 983-987, 1986.

[47] S. W. Lichtman, K. Pisarska, E. R. Berman et al., "Discrepancy between self-reported and actual caloric intake and exercise in obese subjects," The New England Journal of Medicine, vol. 327, no. 27, pp. 1893-1898, 1992.

[48] A. E. Black, A. M. Prentice, G. R. Goldberg et al., "Measurements of total energy expenditure provide insights into the validity of dietary measurements of energy intake," Journal of the American Dietetic Association, vol. 93, no. 5, pp. 572-579, 1993.

[49] S. Z. Yanovski and N. G. Sebring, "Recorded food intake of obese women with binge eating disorder before and after weight loss," International Journal of Eating Disorders, vol. 15, no. 2, pp. 135-150, 1994.

[50] K. Westerterp and A. H. C. Goris, "Validity of the assessment of dietary intake: problems of misreporting," Current Opinion in Clinical Nutrition and Metabolic Care, vol. 5, no. 5, pp. 489493, 2002.

[51] F. B. Scagliusi, E. Ferriolli, K. Pfrimer et al., "Characteristics of women who frequently under report their energy intake: a doubly labelled water study," European Journal of Clinical Nutrition, vol. 63, no. 10, pp. 1192-1199, 2009. 


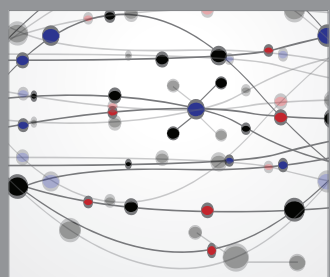

The Scientific World Journal
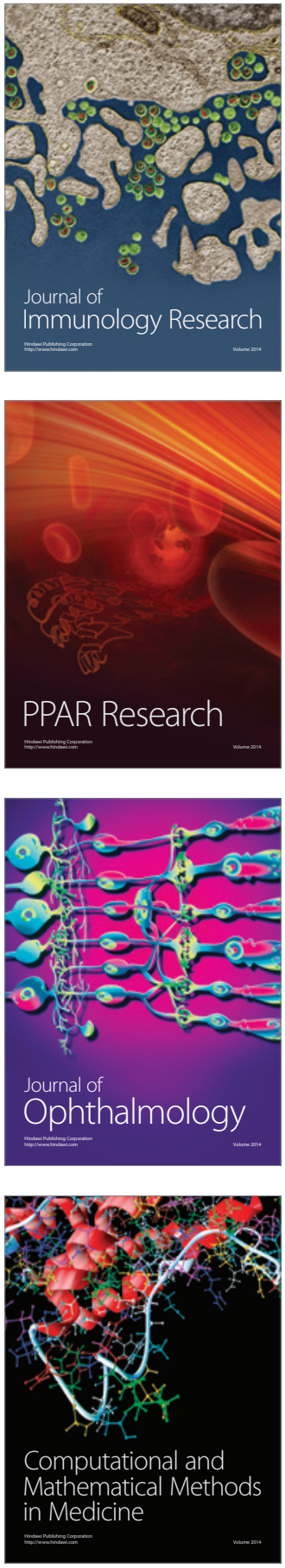

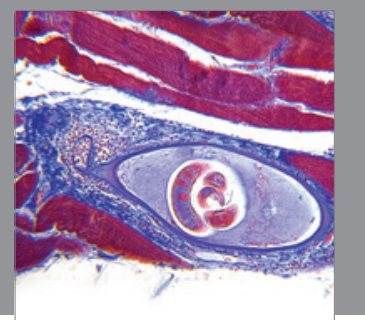

Gastroenterology

Research and Practice
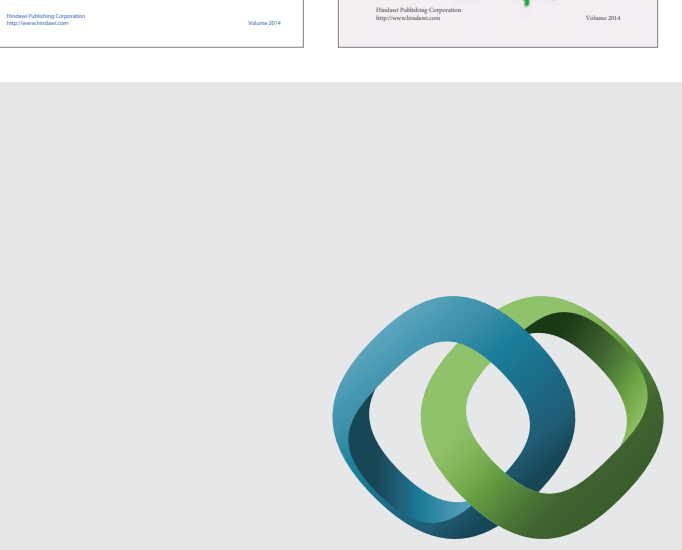

\section{Hindawi}

Submit your manuscripts at

http://www.hindawi.com
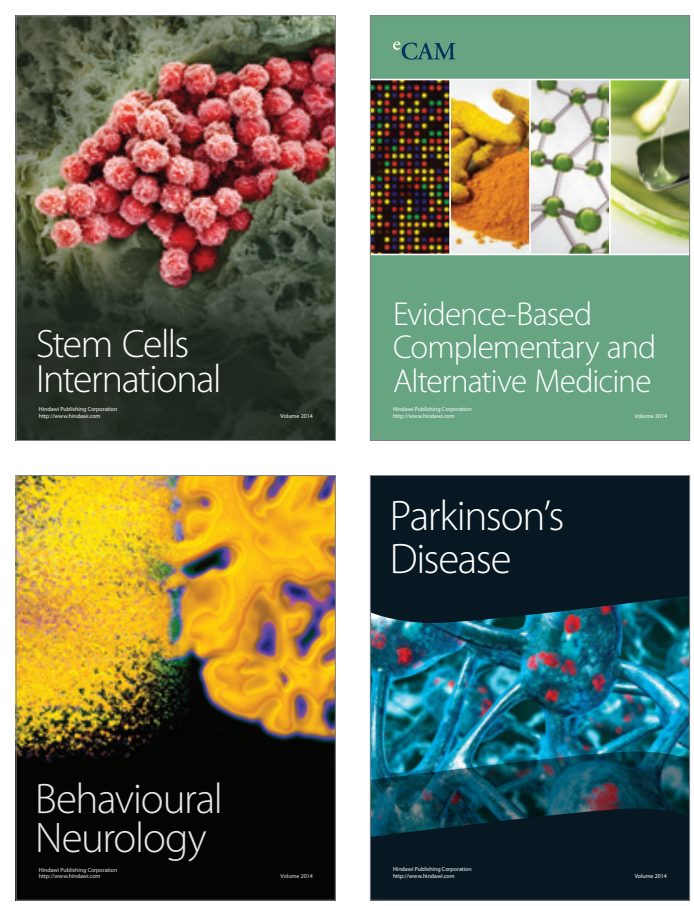

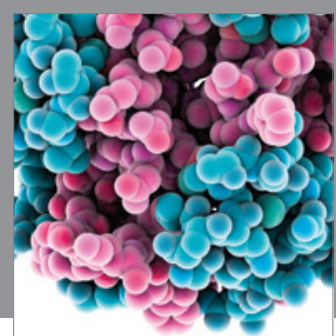

Journal of
Diabetes Research

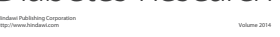

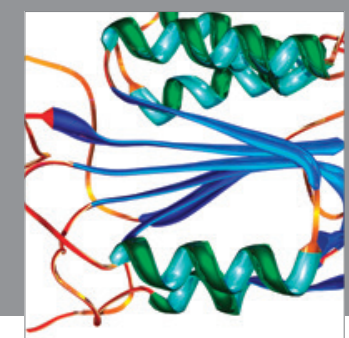

Disease Markers
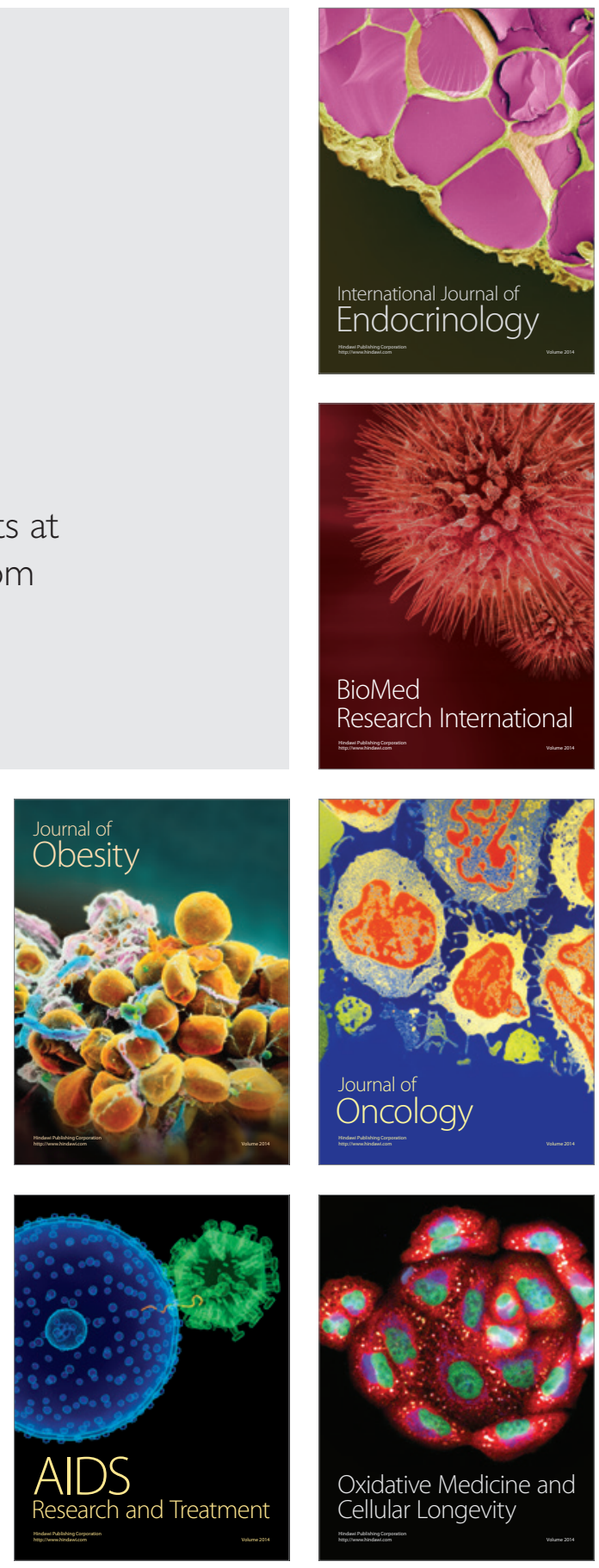